\title{
Clinical Events Start Timepoint
}

National Cancer Institute

\section{Source}

National Cancer Institute. Clinical Events Start Timepoint. NCI Thesaurus. Code C87860.

A point in time that indicates the beginning of a clinical event. 